\title{
MRI-guided Wire Localization Open Biopsy is Safe and Effective for Suspicious Cancer on Breast MRI
}

\author{
Hai-Yi Wang ${ }^{1,2}$, Yu-Nian Zhao ${ }^{3}$, Jian-Zhong Wu u $^{4}$ Zheng Wang ${ }^{2}$, Jing-Hai Tang ${ }^{1 *}$
}

\begin{abstract}
Background: Magnetic resonance imaging of breast, reported to be a high sensitivity of $94 \%$ to $100 \%$, is the most sensitive method for detection of breast cancer. The purpose of this study was to investigate our clinical experience in MRI-guided breast lesion wire localization in Chinese women. Materials and Methods: A total of 44 patients with 46 lesions undergoing MRI-guided breast lesion localization were prospectively entered into this study between November 2013 and September 2014. Samples were collected using a 1.5-T magnet with a special MR biopsy positioning frame device. We evaluated clinical lesion characteristics on pre-biopsy MRI, pathologic results, and dynamic curve type baseline analysis. Results: Of the total of 46 wire localization excision biopsied lesions carried out in 44 female patients, pathology revealed fourteen malignancies $(14 / 46,30.4 \%)$ and thirty-two benign lesions $(32 / 46,69.6 \%)$. All lesions were successfully localized followed by excision biopsy and assessed for morphologic features highly suggestive of malignancy according to the American College of Radiology Breast Imaging Reporting and Data System (BI-RADS) category of MRI (C4a=18, C4b=17, C4c=8,C5=3). Of 46 lesions, 37 were masses and 9 were non-mass enhancement lesions. Thirty-two lesions showed a continuous kinetics curve, 11 were plateau and 3 were washout. Conclusions: Our study showed success in MRI-guided breast lesion wire localization with a satisfactory cancer diagnosis rate of $30.4 \%$. MRI-guided wire localization breast lesion open biopsy is a safe and effective tool for the workup of suspicious lesions seen on breast MRI alone without major complications. This may contribute to increasing the diagnosis rate of early breast cancer and improve the prognosis in Chinese women.
\end{abstract}

Keywords: Breast cancer - MRI-guided - wire localization - excision biopsy

Asian Pac J Cancer Prev, 16 (5), 1715-1718

\section{Introduction}

Magnetic resonance imaging (MRI) of the breast, reported to be a high sensitivity of $94 \%$ to $100 \%$, is the most sensitive technique for the detection of breast cancer.Given these benefits, MRI holds promise for detectingcancers occult on other imaging, such as mammography and ultrasonography. Although MRI has a high sensitivity and negative predictive value in detecting breast lesions, its specificity is comparatively low, ranging from $37 \%$ to $72 \%$ (Saslow et al., 2007). As a consequence, it also detects indeterminate lesions required biopsy or surgery for definitive pathological diagnosis, which the breast lesions are seen only on MRI.A MRI-guided tissue sampling method, such as MRI-guided breast lesion wire localization orpercutaneous core biopsy, is the technique of choice for lesions that are visible only on breast MRI, and it has been performed successfully for many years in the western countries (Gebauer et al., 2006; Ghate et al., 2006; Han et al., 2008). Except for the recently published preliminary experience with MRI-guided breast lesion localization and vacuum-assisted breast biopsy in Japan and Korea (Tozaki et al., 2007; Tozaki et al., 2009; Tozaki et al., 2010), there are no published data in Chinese women. The aim of this retrospective study was therefore to evaluate our initial clinical experience with MRI-guided of breast lesions wire localization biopsy, which was visible only on MRI in Chinese women. We report our initial clinical experience of 46 wire localization excision biopsied lesions carried out in 44 female patientsin Chinese women.

\section{Materials and Methods}

\section{Patient population}

This was a retrospective study for assessing the usefulness of MRI-guided breast lesion wire localization, approved by the Institutional Review Board of our hospital. Informed consent was obtained from all patients. A total of 44 patients with 46 lesions scheduled for MRIguided breast lesion wire localization and surgical excision biopsy between November 2013 and September 2014

${ }^{1}$ Department of General Surgery, Nanjing Medical University Affiliated Cancer Hospital, Cancer Institute of Jiangsu Province, ${ }^{3}$ Radiology Department, ${ }^{4}$ Research Center of Clinical Oncology, the Affiliated Jiangsu Cancer Hospital, Nanjing Medical University, Nanjing, ${ }^{2}$ Department of Gland and Vascular Surgery, Tongling People’s Hospital, Anhui, China *For correspondence: jhtangnj@ yeah.net 
were enrolled to participate in this retrospective study. The patients ranged in age from 26 to 67 years and the median age was 48.4 years.

\section{Breast MRI equipment and technique for breast lesions wire localization}

At our hospital, MRI scanner was performed by using a 1.5-T MR imaging unit (Achieva; Philips Medical Systems, Best, the Netherlands)with the patient prone and the breast positioned within a dedicated surface breast coil with seven-channeland compressed within a grid.The MRI images with the Achieve scanner were acquired using the following sequences: sagittal, fatsuppressed, and fast spin-echo T2-weighted imaging sequence (Repetition time/echo time 6,000/100 ms, flip angle $12^{\circ}, 30$ slices, field of view of $340 \times 340 \mathrm{~mm}$, matrix $340 \times 340$, number of excitations of $1 \mathrm{~mm}$ slice thickness with no gap ) and pre-contrast and post-contrast dynamic axial T1-weighted three-dimensional, fat-suppressed, fat-spoiled gradient-echo sequence (Repetition time/echo time 6.9/3.4, flip angle of $12^{\circ}, 1.0 \mathrm{~mm}$ slice thickness with no gap, acquisition time of 1 minute 31 seconds). A T1weighted high-resolution isotropic volume examination (THRIVE) 3D gradient-echo sequence with active fat suppression was performed to find the enhancement lesion.The imaging parameters were as follows: TR/ TE, 5.1/2.5; flip angle, $12^{\circ}$; FOV, $340 \times 340 \mathrm{~mm}$; matrix, $340 \times 340$; section thickness, $1.0 \mathrm{~mm}$.Before the patient is positioned, informed consent is obtained. The images were obtained before and after a rapid bolus injection of gadolinium- diethylenetriamine pent acetic acid (Magnevist; Schering, Berlin, Germany) at $0.2 \mathrm{mmol} / \mathrm{kg}$ of body weight, delivered through an indwelling intravenous catheter followed by a 10-ml saline flush, which was also administered at a rate of $2 \mathrm{~mL} / \mathrm{s}$. Once the lesion is localized, an MR-compatible guide needle is introduced to the appropriate depth. After imaging confirms appropriate location and depth, a guide wire is deployed through the needle. Before the excision biopsy is performed, the breast images are reviewed to localize the lesion based on the sagittal and axial planes provided by MRI to predict the location.After the localization procedure, a two-view targeted mammogram is usually obtained, in terms of clock position, depth, distance from the nipple, surrounding breast tissue, and noted landmarks, so that the surgeon can see the location of the wire and relation- ship to the nipple and breast tissue.

Two experienced radiologists, who was informed about the patients' clinical course and imaging history such as mammograms and mammography examinations of the breasts, interpreted the imaging findings according to the American College of Radiology Breast Imaging Reporting and Data System (BI-RADS) categories for MRI (American College of Radiology, 2003). All lesions were classified into category 4 or category 5 on MRI. For all cases, the following lesion characteristics were recorded: type (mass or non-mass enhancement), and dynamic curve type baseline analysis. If a lesion is found on MRI, the patients' recent mammogram is reviewed to ensure that there are no correlative calcifications or masses that could potentially be targeted for biopsy using stereotactic guidance.

\section{Data analysis}

Data collection,including histopathological results, BI-RADS, enhancement pattern and dynamic curve type baseline analysis for breast MRI -guided wire localization,was performed by reviewing medical records.

\section{Results}

MRI-guided wire localization was all successfully performed in 44 of 46 lesions $(100 \%, 46 / 46)$. Histopathological results of theMRI-guided wire localization and lesion characteristics on pre-biopsy MRI are summarized in Table 1.All lesions had morphologic features suspicious or highly suggestive of malignancy according to the BI-RADS category of MRI $(\mathrm{C} 4 \mathrm{a}=18$, $\mathrm{C} 4 \mathrm{~b}=17, \mathrm{C} 4 \mathrm{c}=8, \mathrm{C} 5=3$ ).

Of the 46 enhancedbreast lesions identified in 44 women, $14(30.4 \%)$ were malignant and $32(69.6 \%)$ were benign. Among the cases of confirmed malignancies, 7 $(15.2 \%)$ were ductal carcinoma in situ (DCIS) and other $7(15.2 \%)$ were invasive ductal carcinoma (IDC). Of the 32 benign lesions, $20(43.5 \%)$ were breast adenosis, 10 $(21.7 \%)$ were epithelial hyperplasia $(\mathrm{EH})$ and $2(4.4 \%)$

Table 1. Clinical Lesion Characteristics on Prebiopsy MRI, and Pathologic Results in 46 Lesions

\begin{tabular}{|c|c|c|c|c|c|c|}
\hline & \multicolumn{3}{|c|}{ Benign } & \multicolumn{3}{|c|}{ Malignant } \\
\hline & Adenosis & $\mathrm{EH}$ & $\overline{\mathrm{ADH}}$ & $\overline{\text { DCIS }}$ & IDC & $\overline{\text { total }}$ \\
\hline Number(percentage) & $20(43.5 \%)$ & $10(21.7 \%)$ & $2(4.4 \%)$ & $7(15.2 \%)$ & $7(15.2 \%)$ & $46(100 \%)$ \\
\hline \multicolumn{7}{|l|}{ BI-RADS } \\
\hline $4 \mathrm{~A}$ & $13(28.2 \%)$ & $5(10.9 \%)$ & 0 & 0 & 0 & $18(39.1 \%)$ \\
\hline $4 \mathrm{~B}$ & $4(8.7 \%)$ & $2(4.3 \%)$ & $2(4.3 \%)$ & $5(10.9 \%)$ & $4(8.7 \%)$ & $17(37.0 \%)$ \\
\hline $4 \mathrm{C}$ & $3(6.5 \%)$ & $3(6.5 \%)$ & 0 & $1(2.2 \%)$ & $1(2.2 \%)$ & $8(17.4 \%)$ \\
\hline 5 & 0 & 0 & 0 & $1(2.2 \%)$ & $2(4.3 \%)$ & $3(6.5 \%)$ \\
\hline \multicolumn{7}{|l|}{ enhancement pattern } \\
\hline mass enhancement & $19(41.3 \%)$ & $8(17.4 \%)$ & $2(4.3 \%)$ & $3(6.5 \%)$ & $5(10.9 \%)$ & $37(80.4 \%)$ \\
\hline non-mass enhancement & $1(2.2 \%)$ & $2(4.3 \%)$ & 0 & $4(8.7 \%)$ & $2(4.3 \%)$ & $9(19.6 \%)$ \\
\hline \multicolumn{7}{|l|}{ kinetics } \\
\hline continuous & $15(32.6 \%)$ & $7(15.2 \%)$ & $1(2.2 \%)$ & $5(10.9 \%)$ & $4(8.7 \%)$ & $32(69.6 \%)$ \\
\hline plateau & $5(10.9 \%)$ & $3(6.5 \%)$ & $1(2.2 \%)$ & $1(2.2 \%)$ & $1(2.2 \%)$ & $11(23.9 \%)$ \\
\hline washout & 0 & 0 & 0 & $1(2.2 \%)$ & $2(4.3 \%)$ & $3(6.5 \%)$ \\
\hline
\end{tabular}

*MRI magnetic resonance imaging,EH epithelial hyperplasia,ADH atypical ductal hyperplasia, DCIS ductal carcinoma in situ, IDC invasive ductal carcinoma, BI-RADS Breast Imaging Reporting and Data Systems 
were atypical ductal hyperplasia (ADH).

According the type of imaging, the detected lesions included 37 masses and nine non-masslike enhancements. Of the 37 masslikeenhancement lesions, 19 (95\%) were breast adenosis, $8(80 \%)$ were $\mathrm{EH}, 2$ (100\%) were $\mathrm{ADH}$, $3(42.9 \%)$ were DCIS and $5(71.4 \%)$ were IDC.In addition, the frequency of non-masslike enhancement was higher in malignancy $(66.7 \%, \mathrm{n}=6)$ than in benign $(33.3 \%, \mathrm{n}=3)$.

The detection of the enhanced lesions for a curve showing continuous type was $69.6 \%$ (32/46 [benign, $\mathrm{n}=23$; malignancy, $\mathrm{n}=9]$ ). The detection of the enhanced lesions for a curve showing plateau type was $23.9 \%$ (11/46 [benign, $n=9$; malignancy, $n=2]$ ). The detection of the
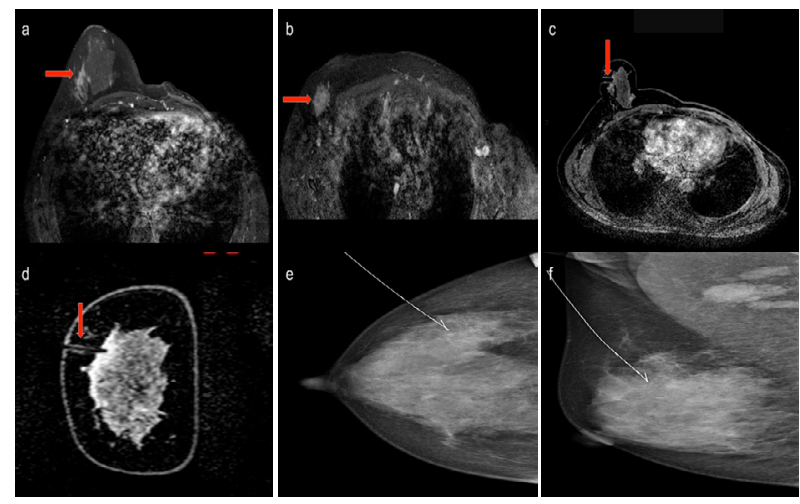

Figure 1. A 44-Year-Old Woman with a Diagnosis of Left Breast Cancer in 8 Years Ago. (a) An only-MRIvisible BI-RADS 4C lesion of the contralateral breast (red arrow) was revealed an invasive ductal carcinoma after MRI-guided. (b) The right axillary lymph nodes was tumescent found as first symptom (red arrow), which could be shown in ultrasound and mammography. (c) and (d) The axial and coronal image of the right occult lesion after MRI guided wire localization. (e) and (f)The cranio-caudal view and medio-lateral oblique view of the mammography pre-operation image after MRI guided wire localization, which lead to the focus anatomy lesion position for excision biopsy
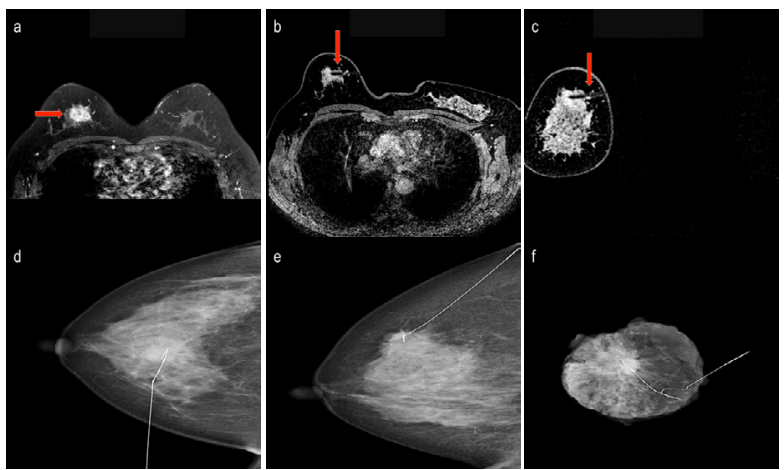

Figure 2. A 48-Year-Old Woman with Structural Disorder Only in the upper Quadrant Breast Showed by Mammography, and MRI-guided Biopsy was Recommended. (a) Images obtained during MR-guided biopsy showed irregular enhancing mass in the upper quadrant breast(red arrow). (b) and (c) The axial and coronal image of the right occult lesion after MRI guided wire localization( red arrow). (d) and (e) The cranio-caudal view and medio-lateral oblique view of the mammography image is performed before biopsy. (f) The biopsy specimen imaging was showed by mammography after excision biopsy. The histology revealed an invasive ductal carcinoma after MRI-guided
MRI-guided Wire Localization Open Biopsy Based on Breast MRI enhanced lesions for a curve showing washout type was $6.5 \%$ (3/46 [benign, $n=0 ;$ malignancy, $n=3]$ ).

The dynamic curve type baseline analysis of all the benign lesions $(32 / 46,69.6 \%)$ were continuous pattern or plateau pattern, however the washout pattern was described only by malignant lesions $(6 / 46,13.0 \%)$.

\section{Discussion}

Breast cancer incidence appears to be increasing in Chinese women and Chinese people have experienced rapid socioeconomic changes, especially in large cities (Deng et al., 2013; Liu et al., 2013; Sun et al., 2013). Conventionally, almost all mammary lesions are detected by anultrasound and amammography. However, a small part of lesions cannot be shown by only a magnetic resonance imaging (MRI). Due to the superior sensitivity of breast MRI as high as 94\%-100\%,MRI-guided wire localization breast biopsy is a very useful means for the pathological diagnosis of these occult lesions. Moreover, because of its lower specificity, ranging from 37\%-97\%, it also detects additional lesions that may be benign by require biopsy for definitive diagnosis.

This study reports our initial experience with MRIguided excisional biopsy. 44 patients with the 46 lesions were performed by MRI-guided wire localization breast biopsy to seen only by MRI. These biopsied specimens resulted benign lesions in 32 and malignant lesions in 14 . Compared with previous reports of studies on MRI-guided breast excisional biopsy, our study included a higher technical success rate (46 out of 46,100\%), which has not been reported previously (Heywang et al., 1999; Lehman et al., 2005; Liberman et al., 2005; Perlet et al., 2006).

It is necessary for an efficient and successful biopsy to plan and prepare carefully. When an occult or suspicious mass is seen only on MRI, the breast MR images are reviewed to localize the lesion based on the sagittal and axial planes provided by MRI to predict the location. And then targeted mammography is performed, to make careful correlation between the MRI and mammography finding. On gaining experience, it is essential to note the location of the lesion in terms of clock position, depth, distance from the nipple, surrounding breast tissue, and landmarks on mammography. It can have a hightechnique success, which has not beenreported in previous study. Moreover, it is important to understand the differences in breast positioning and the effect that will have on lesion location, which is useful for surgeon to operate the excision biopsy.

The chances of finding an indeterminate mass have been reported to be between $23 \%$ and $89 \%$ about targeted ultrasound or mammography (Beran et al., 2005; Meissnitzer et al., 2009; Abe et al., 2010). The probability of finding an ultrasound or mammography correlate is higher for masses rather than nonmasslike enhancement, for larger lesions, and for BIRADS 5 lesions. However,for all indeterminate lesions, the accuracy of the MRI is almost perfect, especially on nonmasslike enhancement lesions in our study.

According to the previous study, Lee et al. (2007) reported that $7 \%$ of MRI-guided biopsies were inconformity and $30 \%$ of those were malignant, and Ductal Carcinoma 
in Situ (DCIS) was upgraded to an invasive cancer in $17 \%$ of the cases following MRI-guided biopsy .Besides, Liberman et al. (2007) founded a 38\% underestimation rate in cases of atypical ductal hyperplasia found on MRIguided biopsy. Therefore, if the result is benign, a 6-month follow-up MRI is reasonable recommended.

The Postbiopsy complications,such as significant bleeding, infection, vasovagal reaction, contralateral skin piercing, were not observed.

With an increase of the opportunity of MRI for the mammary lesions, these lesions become increasingly large as expected. And this biopsy may contribute to increase the diagnosis rate of occult or early breast cancer, and the prognosis is indeeed improved in Chinese women.

Our study had some limitations, and, as such, we emphasize that this is aninitial study. The first limitation of the study is the small sample size. Second, we did not correlate with cancer stage at diagnosis, which might influence imaging features.Third,we did not correlate the hormonal status of the patient and the lesion visibility on MRI.Fourth, is there an imaging-histologic correlation, or do the biopsy results sufficiently explain the imaging findings?As a consequence, long-term follow-up MRI is strongly recommended to the high risk patients. Finally, the samples wasn't involved in small volume breast.The requirements for wire localization of the compressed breast is more than $25 \mathrm{~mm}$, however, the thickness of most Chinese women is less than $25 \mathrm{~mm}$ after fixed and compressed. Therefore, small volume breast cannot meet the needs of the MRI-guided wire localization biopsy device.

In conclusion, in our initial study, we have identified that MRI-guided breast lesion wire localization is safely, easily and effectively performed for obtaining occult breast lesions without major complications. During our initial experience a success rate of $100 \%$ was achieved, which was quite satisfactory. MRI-guided wire localization breast biopsy may contribute to the early diagnosis of breast cancer patients in China. MRI-guided biopsy is the only method that can confirm suspicious enhancing breast lesions in these patients, although it does pose some challenges.And we believe that MRI-guided wire localization breast biopsy will be an important diagnostic modality with careful preparation, proper positioning, and directional localization in Chinese women. Furthermore ,continued work will address the use of the early diagnosis of the small volume breast cancer avoid penetrating into the breast, as well as the use ofMRI-guidedvacuumassisted breast biopsy in China.

\section{Acknowledgements}

This work was supported by the Natural Science Foundation of China provided to Jin-hai Tang (81272470).

\section{References}

Abe H, Schmidt RA, Shah RN, et al (2010). MR-directed ("Second-Look") ultrasound examination for breast lesions detected initially on MRI:MR and sonographicfindings. Am J Roentgenol, 194, 370-7.
American Collegeof Radiology (2003). BI-RADSbreast imaging and reporting system:breast imaging atlas. reston, VA: American college of radiology.

Beran L, Liang W, Nims T, et al (2005). Correlation of targeted ultrasound with magnetic resonance imaging abnormalities of the breast. Am J Surg, 190, 592-4.

Deng QQ, Huang XE, Ye LH, et al (2013). Phase II trial of Loubo $^{\circledast}$ (Lobaplatin) and pemetrexed for patients with metastatic breast cancer not responding to anthracycline or taxanes. Asian Pac J Cancer Prev, 14, 413-7.

Gebauer B, Bostanjoglo M, Moesta KT, et al (2006). Magnetic resonance-guided biopsy of suspicious breast lesions with a handheld vacuum biopsy device. Acta Radiol, 47, 907-13.

Ghate SV, Rosen EL, Soo MS, et al (2006). MRI-guided vacuumassisted breast biopsy with a handheld portable biopsy system. AJR Am J Roentgenol, 186, 1733-6.

Han BK, Schnall MD, Orel SG, et al (2008). Outcome of MRIguided breast biopsy. AJR Am J Roentgenol, 191, 1798-804.

Heywang-Kobrunner SH, Heinig A, Schaumloffel U, et al (1999). MR-guided percutaneous excisional and incisional biopsy of breast lesions. Eur Radiol, 9, 1656-65.

Lee JM, Kaplan JB, Murray MP, et al (2007). Imaging histologic discordance at MRI-guided 9-gauge vacuum assisted breast biopsy. Am J Roentgenol, 189, 852-9.

Lehman CD, Deperi ER, Peacock S, et al (2005). Clinical experience with MRI-guided vacuum-assisted breast biopsy. AJR Am J Roentgenol, 184, 1782-7.

Liberman L, Bracero N, Morris E, et al (2005). MRI-guided 9-gauge vacuum-assisted breast biopsy: initial clinical experience. AJR Am J Roentgeno, 185, 183-93.

Liberman L, Holland AE, Marjan D, et al (2007). Underestimation of atypical ductal hyperplasia at MRI-guided 9-gauge vacuum-assisted breast biopsy. Am J Roentgenol, 188, 684-90.

Liu YC, Zhou SB, Gao F, et al (2013). Phase II study on breast conservative surgery plus chemo- and radiotherapy in treating Chinese patients with early staged breast cancer. Asian Pac J Cancer Prev, 14, 3747-50.

Meissnitzer M, Dershaw DD, Lee CH, et al (2009). Targeted ultrasound of the breast in women with abnormal MRIfindings for whom biopsy has been recommended. Am J Roentgenol, 193, 1025-9.

Perlet C, Heywang-Kobrunner SH, Heinig A, et al (2006). Magnetic resonance-guided, vacuum-assisted breast biopsy: results from a European multicenter study of 538 lesions. Cancer, 106, 982-90.

Sun MQ, Meng AF, Huang XE, et al (2013). Comparison of psychological influence on breast cancer patients between breast-conserving surgery and modified radical mastectomy. Asian Pac J Cancer Prev, 14, 149-52.

Tozaki M, Yamashiro N, Fukuma E (2007). MR-guided vacuumassisted breast biopsy using a non-titanium needle. Magn Reson Med Sci, 6, 259-64.

Tozaki M, Yamashiro N, Sakamoto M, et al (2010). Magnetic resonance-guided vacuum-assisted breast biopsy: results in 100 Japanese women. Jpn J Radiol, 28, 527-33.

Tozaki M, Yamashiro N, Suzuki T, et al (2009). MR-guided vacuum-assisted breast biopsy: is it an essential technique? Breast Cancer, 16, 121-5.

Saslow D, Boetes C, Burke W, et al (2007). American cancer society guidelines for breast screening with MRI as an adjunct to mam- mography. CA Cancer J Clin, 57, 75-89. 